\title{
Phase Diagrams of the Penson-Kolb-Hubbard Model with Repulsive Pair-Hopping Interaction
}

\author{
W.R. CzART* AND S. RobaszKIEWICZ \\ Electron States of Solids Division, Faculty of Physics, Adam Mickiewicz University, \\ Umultowska 85, 61-614 Poznań, Poland
}

\begin{abstract}
We study the extended Hubbard model with on-site density-density $U$ and intersite pair hopping $J$ interactions, i.e. the Penson-Kolb-Hubbard model. This report focuses mainly on the properties of the model at $T \geq 0$ in the case of repulsive $J(J<0)$ which may stabilize superconductivity with $\eta$-pairing. The analysis is performed within the (broken symmetry) Hartree-Fock approximation for arbitrary interaction parameters $(J<0$ and $U)$ and electron concentration $(0<n<2)$ on the $d=2$ square lattice. The phase diagrams of the model at $T=0$ and at finite temperatures are examined taking into account magnetic and charge-ordered phases and superconducting states with $\eta$ - and $s$-wave pairing.
\end{abstract}

DOI: 10.12693/APhysPolA.130.613

PACS/topics: 74.20.-z, 71.28.+d, 74.20.Mn

\section{Introduction}

The Penson-Kolb-Hubbard (PKH) model is one of the conceptually simplest models for studying superconductivity with short coherence length and for description of various superconducting, magnetic and other electron orderings in narrow band systems [1-14]. Its Hamiltonian has the form

$$
\begin{aligned}
H & =-\sum_{i j \sigma} t_{i j}\left(c_{i \sigma}^{+} c_{j \sigma}+\text { H.c. }\right)-\mu \sum_{i \sigma} c_{i \sigma}^{+} c_{i \sigma} \\
& +U \sum_{i} n_{i \uparrow} n_{i \downarrow}-\frac{1}{2} J \sum_{\langle i j\rangle}\left(c_{i \uparrow}^{+} c_{i \downarrow}^{+} c_{j \downarrow} c_{j \uparrow}+\text { H.c. }\right),
\end{aligned}
$$

where $n_{i \sigma}=c_{i \sigma}^{+} c_{i \sigma}, t_{i j}$ is the single electron hopping integral, $U$ is the on-site density-density interaction, $J$ is the pair hopping (intersite charge exchange) interaction, $\mu$ is the chemical potential. $\langle i j\rangle$ restricts the sum to nearest neighbors (nn) and $n=\frac{1}{N} \sum_{i \sigma}\left\langle n_{i \sigma}\right\rangle$.

In general, the model includes two pairing mechanisms: (i) nonlocal pairing mechanism (the intersite pair hopping term $J$ ) and (ii) the on-site interaction $U$, if $U<0$. In the following we will focus mainly on the case of repulsive $J(J<0)$ which can stabilize superconducting $\eta$ pairing order, i.e. the state with the Cooper-pair centerof-mass momentum $\boldsymbol{q}=\boldsymbol{Q}\left(\boldsymbol{Q}=\frac{\Pi}{a}, \frac{\Pi}{a}, \ldots,\right)$. We will discuss the stability of the homogeneous superconducting states including $s$-wave (S) and $\eta$-pairing (eta) as well as the magnetic (antiferromagnetic (AF) and ferromagnetic $(\mathrm{F})$ ) and charge-ordered $(\mathrm{CO})$ phases in the considered system. The analysis is performed at $T=0$ and for finite temperatures. In the case of superconducting states the conditions for the crossover to the Bose-Einstein condensation (BEC) regimes are derived. Some preliminary results concerning the $T=0$ phase diagrams have been presented in Ref. [9] for $J<0$ and in Ref. [8] for $J>0$.

\footnotetext{
*corresponding author; e-mail: czart@amu.edu.pl
}

\section{Results and discussion}

As in Ref. [2] our analysis is based on the (broken symmetry) Hartree-Fock approximation (HFA). In the derivation of the eigensolutions we have assumed an alternate lattice, i.e. $\epsilon_{k+Q}=-\epsilon_{k}$. The stable solutions are determined for arbitrary electron concentration $n$ as the minimum of the free energy of the system $F$ with respect to the variational parameters $x_{\alpha}(\alpha=\mathrm{AF}, \mathrm{F}, \mathrm{CO}, \mathrm{S}, \eta)$, the Fock term $p=1 / 4 N \sum_{k \sigma} \gamma_{k}\left\langle c_{k \sigma}^{+} c_{k \sigma}\right\rangle$ and $\mu$, i.e. by the equations

$$
\frac{\partial F}{\partial x_{\alpha}}=0, \frac{\partial F}{\partial p}=0, \frac{\partial F}{\partial \mu}=0,
$$

from which we get sets of self-consistent equations for each ordering type. The order parameters for considered homogeneous phases are: (i) $\eta$-pairing superconductivity: $x_{\eta}=\frac{1}{N} \sum_{i} \exp \left(\boldsymbol{Q}_{i} \cdot \boldsymbol{R}_{i}\right)\left\langle c_{i \downarrow} c_{\iota \uparrow}\right\rangle$, (ii) $s$-wave pairing superconductivity: $x_{s}=\frac{1}{N} \sum_{i}\left\langle c_{i \downarrow} c_{\iota \uparrow}\right\rangle=\frac{1}{N} \sum_{k}\left\langle c_{-k \downarrow} c_{k \uparrow}\right\rangle$, (iii) charge ordered: $x_{C O}=\frac{1}{2 N} \sum_{i, \sigma} \exp \left(\boldsymbol{Q}_{i} \cdot \boldsymbol{R}_{i}\right)\left\langle c_{i \sigma}^{+} c_{\iota \sigma}\right\rangle$, (iv) ferromagnetic: $x_{F}=\frac{1}{N} \sum_{i, \sigma} \sigma\left\langle c_{i \sigma}^{+} c_{\iota \sigma}\right\rangle$, (v) antiferromagnetic: $x_{A F}=\frac{1}{2 N} \sum_{i, \sigma} \sigma \exp \left(\boldsymbol{Q}_{i} \cdot \boldsymbol{R}_{i}\right)\left\langle c_{i \sigma}^{+} c_{\iota \sigma}\right\rangle$.

The crossovers to the BEC regimes for $\mathrm{S}$ and $\eta$ phases are located after Leggett [15]: from the requirement that $\bar{\mu}$ in superconducting phase reaches the bottom of the electronic band, i.e. from $\bar{\mu}=\mu-U / 2=-B / 2$, where $B=2 z t$ is the bandwidth ( $z$ - the number of $\mathrm{nn})$ and $\bar{\mu}$ for a given phase is determined from the self-consistent Eqs. (2) which are solved at $T=0$.

We have performed analysis of the phase diagrams of the model (1) at $T \geq 0$ for square (SQ) lattice and arbitrary $n(0<n<2)$ involving magnetic orderings for repulsive $U(U>0)$, charge orderings for attractive $U$ $(U<0)$ and the superconducting states for $U>0$ and $U<0$. Due to the electron-hole symmetry of the system the plotted diagrams are symmetric under the transformation $n \rightarrow 2-n$.

The electronic spectrum of the $\eta$-phase consists of two branches $E_{k}^{+}$and $E_{k}^{-}$and the minimum gap between the 
lower and higher band $E_{g}^{\min }=\min E_{k}^{+}-\max E_{k}^{-}$can be either positive or negative, depending on interaction parameters and temperature $[9,16,17]$, which is in contrast with the $s$-wave phase, where $E_{g}>0$ for any $T<T_{c}$.

Figures 1 and 2 present examples of the ground state phase diagrams as a function of interactions and carriers concentration $n$. In the diagrams the transitions to nonordered phase $(\mathrm{N})$ are of the 2nd order, and the transitions between ordered states are of the 1st order. Dashed lines on the figures mark the borders between strong- $\eta$ - and weak- $\eta$-phases (defined later).

In the strong $\eta$ phase at $T=0$ the order parameter $x_{\eta}$ takes its maximum value $x_{\eta}^{\max }=1 / 2 \sqrt{n(2-n)}$ (the same as for $t=0[11-14])$ and $E_{g}^{\min }>0$, while for the weak $\eta$ state $x_{\eta}<x_{\eta}^{\max }$, and $E_{g}^{\min }<0$. For more detailed discussion of these two $\eta$ states see Ref. [9, 16-18]. In addition to the ground state phase boundaries, we denote in the figures the crossovers to the BEC regimes (dasheddotted lines). As we see in definite ranges of interaction parameters the crossover to BEC regimes (both for $s$ wave phase - Fig. 1 and for $\eta$-phase - Figs. 1, 2) can be obtained by changing the electron density.

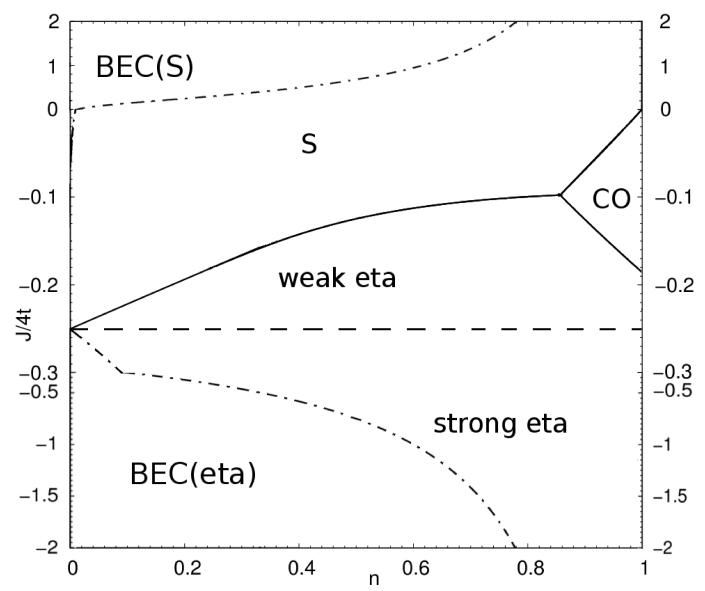

Fig. 1. The ground state phase diagram as a function of pair-hopping interaction $J$ and concentration $n$ for fixed $U / 4 t=-1$. In the diagram, at $J=0$ and $J=-0.3$ there are changes of scale on the $J$ axis. Denotations: $s$-wave superconductivity (S), charge-ordering (CO), $\eta$-pairing state (eta). Dashed line marks the border between strong- and weak- $\eta$ phases, and dash-dotted lines denote crossovers to BEC regimes (SQ lattice).

In Fig. 1 we show the ground state phase diagram of the system as a function of $J$ vs. $n$ plotted for fixed $U / 4 t=-1$. Due to competition between attractive $U$ and repulsive $J$ the charge-ordered phase and $s$-wave pairing superconductivity can be stable in the phase diagram within limited ranges of the model parameters (cf. also Fig. 3 in [9]). The CO phase may occur only close to $n=1$ and at half-filling, while $\mathrm{S}$ phase survives only for small repulsive values of $J$ and obviously for $J>0$. For strong attractive $U$ as that in Fig. 1, the nonordered state $(\mathrm{N})$ does not appear on a diagram. As we can see

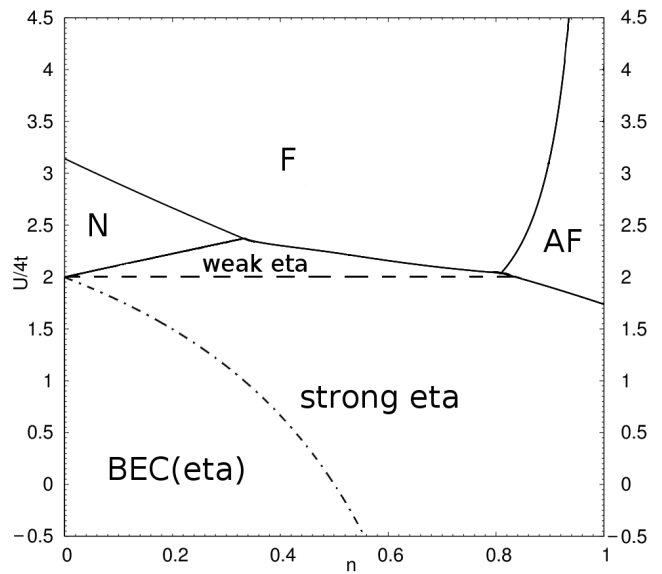

Fig. 2. The ground state phase diagram as a function of $U$ and concentration $n$ for fixed $J / 4 t=-1$. Denotations: nonordered phase $(\mathrm{N})$, ferromagnetism $(\mathrm{F})$, antiferromagnetism (AF). Other denotations as in Fig. 1.

on the plot the $\eta$-phase is stable only below certain critical value $J_{c}<0$ dependent on $n$. Within the $\eta$-phase, there exists a second characteristic value of $J$, which we denote $J_{c 1}$ and $\left|J_{c 1}\right| \geq\left|J_{c}\right|$. For SQ lattice considered in this work $J_{c 1}=-U / z-2 t$ for any $n$. For $|J|>\left|J_{c 1}\right|$ the ground state of the system is characterized by a nonzero gap $E_{g}^{\mathrm{min}}$ and by the order parameter which takes its maximum value $x_{\eta}^{\max }$ (see Fig. 3). In this case the system exhibits the strong $\eta$-pairing phase. On the other hand, weak $\eta$-pairing phase is realized for $\left|J_{c}\right|<|J|<\left|J_{c 1}\right|$ : $E_{g}^{\min }=0$ and $x_{\eta}<x_{\eta}^{\max }$. At $T=0$ the transition between the strong and weak $\eta$-phase takes place at $J=J_{c 1}$ when $E_{g}^{\min }=0$. The range of $n$ of stable weak- $\eta$ phase shrinks with increasing $J(J<0)$ and this phase can exist only for $J<J_{c}<0$.

For large $|J|$ superconducting $\eta$-phase is stable within the whole range of concentration $n(0<n<2)$.

Increasing attractive $U$ increases $\left|J_{c}\right|$ and $\left|J_{c 1}\right|$. In definite ranges of $J$ the crossovers to BEC regimes occur as $n$ is reduced, both for $\eta$ and $s$-wave phases (dash-dotted curves in Fig. 1).

Figure 2 presents the $U$ vs. $n$ phase diagram of the system for fixed $J / 4 t=-1$. For repulsive $U(U>0)$ the $\eta$ pairing competes with magnetic orderings and can be stable only for $U<U_{c}$. In general, the critical value $U_{c}$ depends on $n$, the form of density of states and the value of $J$. Obviously, $U_{c}$ decreases with increasing $|J|$. Within the region of strong- $\eta$ phase stability the crossover to the BEC (eta) regime takes place with decreasing $n$, similarly as in the case shown in Fig. 1.

To clearly demonstrate the nature of the $\eta$-phase induced by pair hopping interaction $J$, we plot in Fig. 3 the ground state results for the case $U=0$. The figures show the $\eta$-phase order parameter $x_{\eta}$ as a function of $n$ for several fixed values of $J / 2 t$ (Fig. $3 \mathrm{a}$ ) and as a function of $J$, for representative values of $n$ (Fig. 3b). In the 

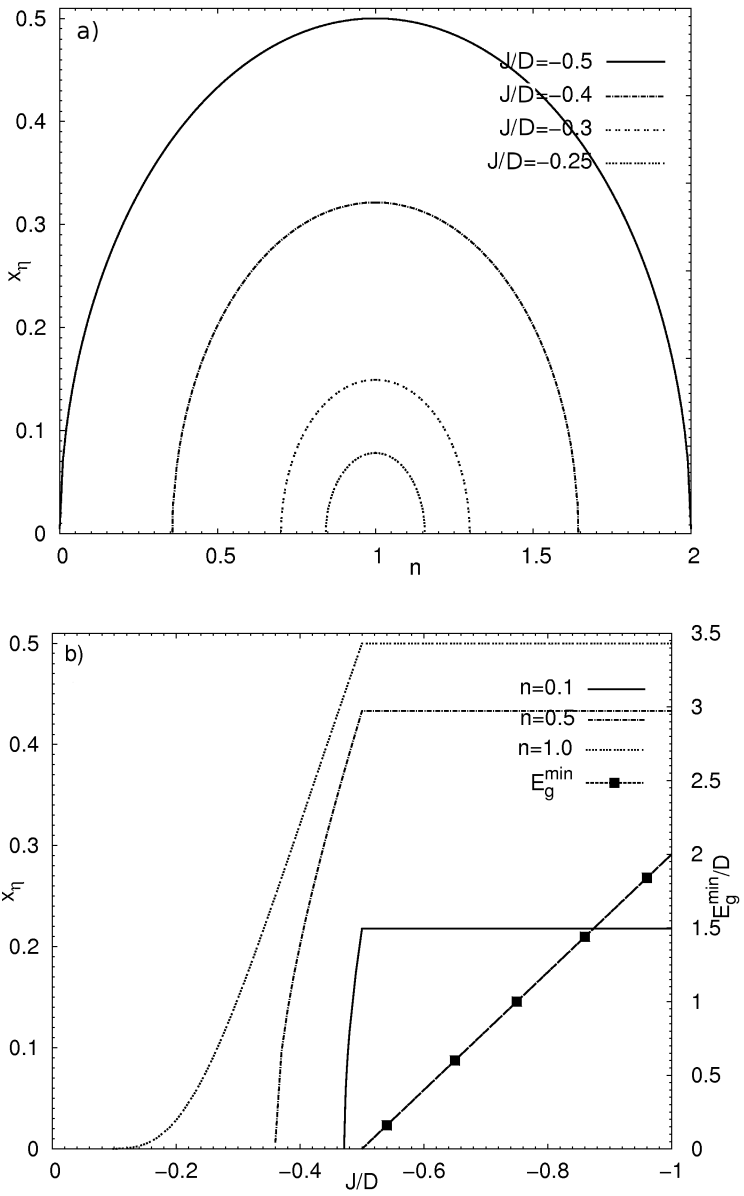

Fig. 3. The ground state plots of (a) the $\eta$-pairing order parameter $x_{\eta}$ as a function of concentration $n$ for fixed values of $J / D: J / D=-0.25 ; J / D=-0.3$; $J / D=-0.4 ; J / D=-0.5$ and (b) the $\eta$-pairing order parameter $x_{\eta}$ and the gap between the lower and higher quasiparticle band $E_{g}^{\mathrm{min}}$ as function of interaction $J$ for fixed values of $n: n=1.0 ; n=0.5 ; n=0.1$. The $E_{g}^{\text {min }}$ versus $J$ line (the same for any $n$ plotted) is denoted with black squares (ם). SQ lattice, $U=0$.

strong $\eta$-phase region $\left(|J| \geq\left|J_{c 1}\right|=2 t\right)$ superconducting $\eta$-phase is stable within the whole range of concentration $n(0<n<2)$. In this regime as we have mentioned earlier $E_{g}^{\min }>0$, and the magnitude of the order parameter $x_{\eta}$ assumes its maximum value $x_{\eta}^{\max }$. In the weak- $\eta$ regime $\left(|J|<\left|J_{c 1}\right|=2 t\right)$ the $\eta$-phase can occur only within a limited range of $n, x_{\eta}<x_{\eta}^{\max }$ and $E_{g}^{\min }<0$. The concentration range of $\eta$-phase stability shrinks with decreasing $|J|$. The magnitude of parameter $x_{\eta}$ decreases with decreasing $|J|$ and $n$. Close to phase boundary with $\mathrm{N}$ phase the $x_{\eta}$ quickly vanishes with $J \rightarrow J_{c}$ and with $n \rightarrow n_{c}$. When the ordered phase sets in for $|J|>\left|J_{c}\right|$, the order parameter $x_{\eta}$ continuously increases till it attains its maximum value (dependent on $n$ ) in the strong- $\eta$ phase.

With increasing $T$ the system can exhibit several different types of behavior depending on the values of in- teraction parameters and $n$. The transitions from $\mathrm{N}$ to ordered phases are of the 2nd order and those between ordered phases are of the 1st order. Examples of the finite temperature phase diagrams for repulsive $U(U>0)$ are plotted in Fig. 4 as a function of concentration $n$ and in Fig. 5 as a function of $U$.

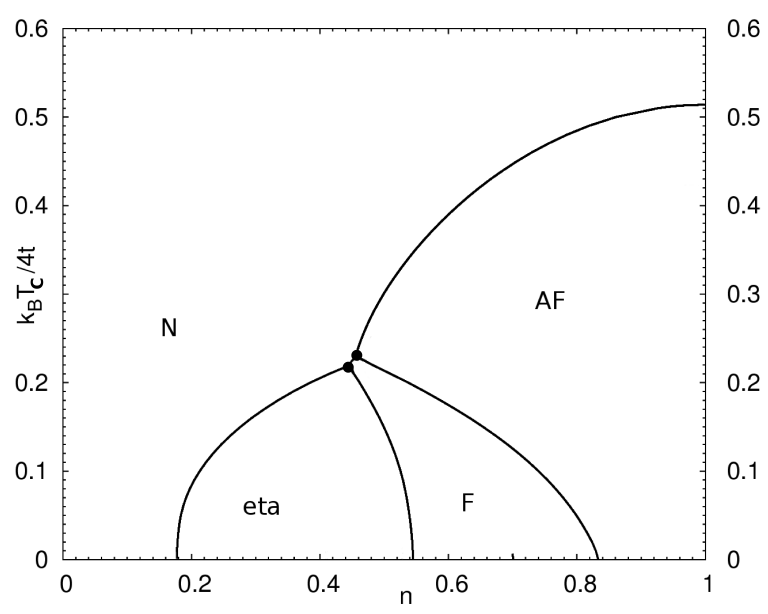

Fig. 4. Finite temperature phase diagram $T$ vs. $n$ plotted for $U / 4 t=2.2$ and $J / 4 t=-1$ for SQ lattice. Filled dots denote the triple points.

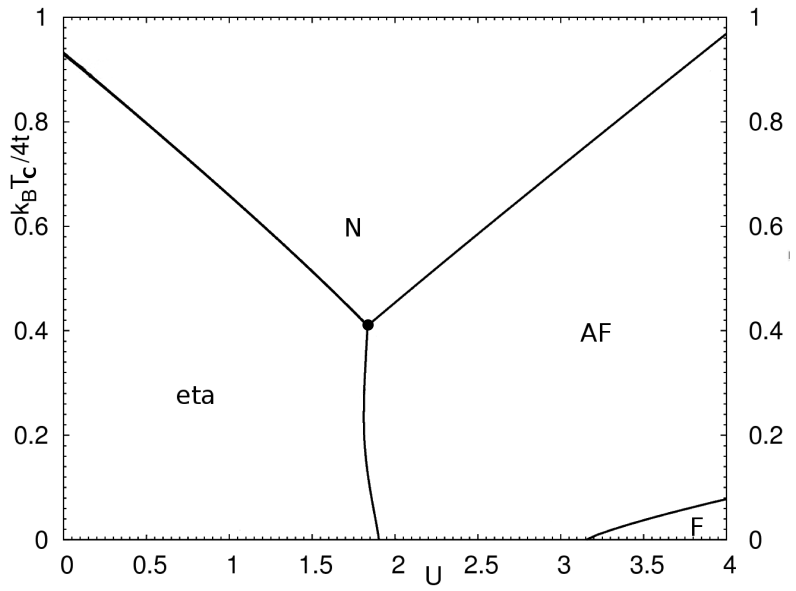

Fig. 5. Finite temperature phase diagram $T$ vs. $U$ plotted for SQ lattice, $n=0.9$ and $J / 4 t=-1$. Filled dot denote the triple point.

In the phase diagram plotted as a function of $n$ (Fig. 4) one can observe the following sequences of phase transitions with increasing $T$ : (i) a single 2 nd order transition $\eta \rightarrow N$, (ii) a sequence of two transitions: 1st order $\eta \rightarrow F, 2$ nd order $F \rightarrow N$, (iii) a sequence of three transitions: two 1st order $\eta \rightarrow F \rightarrow A F$ and 2nd order one $A F \rightarrow N$, (iv) a sequence of two transitions: 1st order $F \rightarrow A F, 2$ nd order $A F \rightarrow N$ and (v) a single 2 nd order transition $A F \rightarrow N$. In our diagram two triple points appear marking transitions between (i) N, $\eta$ and $\mathrm{F}$ phases and (ii) N, F and AF phases. 
The diagram $T$ versus $U$ in the repulsive range of $U$ calculated for fixed values of $J$ and $n$ (Fig. 5) shows that with increasing $T$ the system can exhibit: (i) a single 2nd order transition $\eta \rightarrow N$, (ii) in a limited range of $U$, reentrant $\mathrm{AF}$ phenomenon with a sequence of three transitions: two 1st order $\eta \rightarrow A F \rightarrow \eta$ and one 2nd order $\eta \rightarrow N$, (iii) a sequence of two transitions: 1st order $\eta \rightarrow \mathrm{AF}$ and 2nd order $A F \rightarrow N$, (iv) a single 2nd order transition $A F \rightarrow N$ and finally (v) a sequence of two transitions: 1st order $F \rightarrow A F$ and 2nd order $A F \rightarrow N$. In the diagram a triple point appears as a critical point between $\mathrm{N}, \eta$ and AF phases.

\section{Final remarks}

In the model considered the $\eta$ pairing state can be stable only above some critical values of repulsive $|J|$ dependent on $U$ and $n$. The system in this state never exhibits standard BCS features (cf. also [2, 16-18]). This behaviour is in contrast with the properties of the isotropic $s$-wave state, which for $U \leq 0$ is stable for any attractive $J>0$, and the system exhibits a smooth crossover from the BCS-like limit to the BEC regime with increasing $J[2,6,19-22]$. In the case of $\eta$ pairing we have also found the crossover to BEC regime which can take place in the range of stability of strong- $\eta$ phase as electron density is reduced (cf. Figs. 1 and 2)). Regarding the influence of the on-site interaction $U$, attractive $U(U<0)$ expands the range of stability of $\eta$-phase at $T=0$ towards lower values of $|J|\left(\left|J_{0}\right| \Rightarrow\left|J_{0}\right|+|U|\right)$. The $\eta$-phase can survive also for repulsive values of $U\left(0<U<U_{c}\right)$. Interplay between $U$ and $J$ can stabilize several new phases, magnetic and charge ordered and generate various interesting sequences of phase transitions occurring with increasing temperature.

In this report we have concentrated on the phase diagrams of the model at $T \geq 0$ taking into account only simple types of various homogeneous electron orderings. The analysis of electromagnetic properties of the model and consideration of mixed orderings and phase separated states will be presented elsewhere [16].

\section{Acknowledgments}

We would like to thank R. Micnas and K.J. Kapcia for helpful discussions.

\section{References}

[1] A. Hui, S. Doniach, Phys. Rev. B 48, 2063 (1993).

[2] S. Robaszkiewicz, B. Bułka, Phys. Rev. B 59, 6430 (1999) and references therein.

[3] G.I. Japaridze, A.P. Kampf, M. Sekania, P. Kakashvili, Ph. Brune, Phys. Rev. B 65, 014518 (2001); G.I. Japaridze, E. Müller-Hartmann, J. Phys. Condens. Matter 9, 10509 (1997); G.I. Japaridze, S. Sarkar, Eur. Phys. J. B 27, 139 (2002).
[4] R. Micnas, J. Ranninger, S. Robaszkiewicz, Rev. Mod. Phys. 62, 113 (1990).

[5] S. Robaszkiewicz, W. Czart, Acta Phys. Pol. B 32 3267 (2001).

[6] W. Czart, S. Robaszkiewicz, Phys. Rev. B 64, 104511 (2001).

[7] A. Ptok, K.J. Kapcia, Supercond. Sci. Technol. 28, 045022 (2015); A. Ptok, D. Crivelli, K.J. Kapcia, Supercond. Sci. Technol. 28, 045010 (2015); K.J. Kapcia, W.R. Czart, A. Ptok, J. Phys. Soc. Jpn. 85, 044708 (2016).

[8] W. Czart, S. Robaszkiewicz, Acta Phys. Pol. A 127, 278 (2015).

[9] W. Czart, S. Robaszkiewicz, Acta Phys. Pol. A 127, 275 (2015).

[10] M. Mierzejewski, M. Maśka, Phys. Rev. B 69 054502 (2004); A. Ptok, M. Maśka, M. Mierzejewski, J. Phys. Condens. Matter 21, 295601 (2009); A. Ptok, M.M. Maśka, M. Mierzejewski, Phys. Rev. B 84, 094526 (2011).

[11] K. Kapcia, S. Robaszkiewicz, R. Micnas, J. Phys. Condens. Matter 24, 215601 (2012); K. Kapcia, J. Supercond. Nov. Magn. 27, 913 (2014).

[12] K. Kapcia, S. Robaszkiewicz, J. Phys. Condens. Matter 25, 065603 (2013); K.J. Kapcia, Acta Phys. Pol. A 126, A-53 (2014).

[13] A. Belkasri, F.D. Buzatu, Phys. Rev. B 53, 7171 (1996); F. Dolcini, A. Montorsi, Phys. Rev. B 62, 2315 (2000).

[14] K. Kapcia, Acta Phys. Pol. A 121, 733 (2012); K. Kapcia, J. Supercond. Nov. Magn. 26, 2647 (2013); K.J. Kapcia, J. Supercond. Nov. Magn. 28, 1289 (2015); K.J. Kapcia, Acta Phys. Pol. A 127, 204 (2015).

[15] A.J. Leggett, in: Modern Trends in the Theory of Condensed Matter, Eds. A. Pekalski, J. Przystawa, Springer, Berlin 1980, p. 13.

[16] W. R. Czart, S. Robaszkiewicz, in preparation.

[17] S. Robaszkiewicz, W. Czart, Phys. Status Solidi $B$ 236, 416 (2003); W.R. Czart, S. Robaszkiewicz, B. Tobijaszewska, Phys. Status Solidi B 244, 2327 (2007).

[18] W. Czart, S. Robaszkiewicz, Acta Phys. Pol. A 106 709 (2004).

[19] W.R. Czart, S. Robaszkiewicz, Acta Phys. Pol. A 97, 217 (2000); W. Czart, S. Robaszkiewicz, Acta Phys. Pol. A 100, 885 (2001); A. Ptok, M. Mierzejewski, Acta Phys. Pol. A 114, 885 (2008).

[20] W. Czart, T. Kostyrko, S. Robaszkiewicz, J. Magn. Magn. Mater. 140-144, 2059 (1995).

[21] W. Czart, T. Kostyrko, S. Robaszkiewicz, Physica $C$ 272, 51 (1996).

[22] B. Bułka, S. Robaszkiewicz, Phys. Rev. B 54, 13138 (1996). 